\title{
Design of Ultrasonic Liquid Level Sensor Based on STM32 with MODBUS Protocol
}

\author{
Yanlong $\mathrm{He}^{1,2, \mathrm{a}}$, Changzhe $\mathrm{Wu}^{1, \mathrm{~b}}$, Xiaolin $\mathrm{Huo}^{1, \mathrm{c}}$ \\ ${ }^{1}$ Beijing Key Laboratory of Bioelectromagnetism, Institute of Electrical Engineering, \\ Chinese Academy of Sciences, Beijing 100190, China \\ ${ }^{2}$ University of Chinese Academy of Sciences, Beijing 100190, China \\ aheyanlong@mail.iee.ac.cn, ${ }^{b}$ wuzhe@mail.iee.ac.cn, chuoxl@mail.iee.ac.cn
}

Keywords: Artificial Liver; STM32; Ultrasonic; MODBUS Protocol

Abstract. In the process of blood transfusion in artificial liver, the liquid level of blood inside the dropper must be monitored in real time to ensure the stability and safety of transfusion. According to the requirement of the liquid level detection, we designed a kind of ultrasonic liquid level sensor based on STM32 and MODBUS protocol. Combined with $40 \mathrm{KHz}$ ultrasonic transceiver probes and signal amplification circuits, real-time monitor was implemented by STM32 with its built-in PWM and AD convert modules. The parameters of sensor can be modified by MODBUS commands to adjust the sensitivity, stability and reliability. The sensor had the advantages of simple structure, low cost, high sensitivity. And the experimental results showed that the sensor was applicable to artificial liver systems.

\section{Introduction}

To maintain the balance of water, electrolytes, acid-base and eliminate body toxins, diffusion and convection based on the principle of semipermeable membrane is adopted in the artificial liver system[1].

Liquid level sensor is an indispensable component of artificial liver. Once the liquid level of blood inside the dropper exceed the security level during the treatment process, the sensor will send alarm signal to the host computer. If the alerting signal is not stable and reliable, it may bring unnecessary work to the medical stuffs, cause troubles for hemodialysis and even endanger the life of patients. So, the signal of the sensor must be accurate. In the past, a capacitive sensor was used in artificial liver to detect the liquid level of blood. However, there were several defects. Firstly, the sensitivity of the sensor was inversely proportional to blood flow rate. Secondly, the detection result was susceptible to the surface absorbed water. Thirdly, the installation of capacitive sensor was very complex. To solve the problems and meet the demands of artificial liver, we designed a non-contact detection device. It consists of the $40 \mathrm{KHz}$ ultrasonic transceiver probes and the core controller STM32F103C8T6 with the software function of sensitivity adjustability. The detection will not be affected by light intensity and electromagnetic field in the surrounding. The acquisition and processing of the signal of liquid level is simple, fast and costless [2-4].

\section{System Framework}

The system framework is shown in Fig. 1.

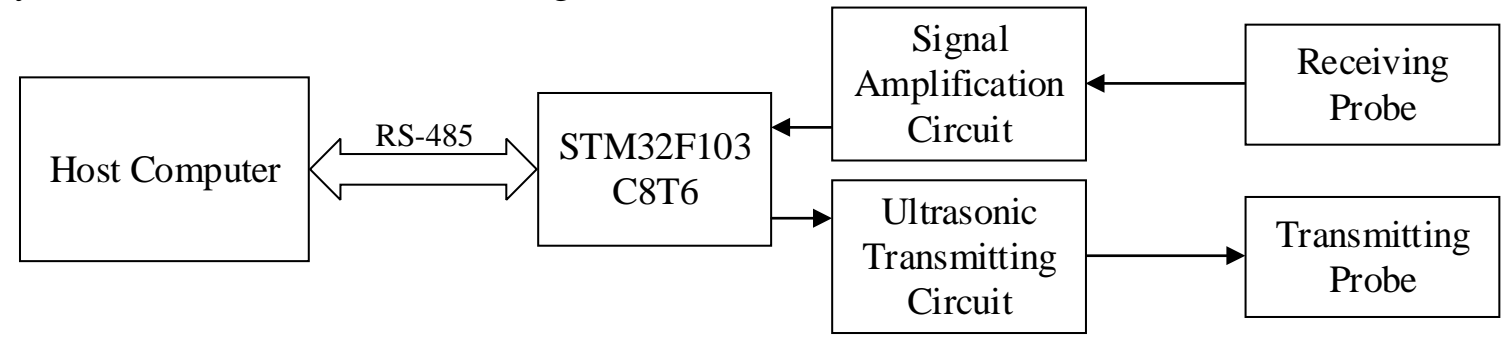

Fig.1. System framework 


\section{Core Controller}

The sensor uses STM32F103C8T6 as the core control unit which is a flash-enhanced microprocessor based on the Cortex-M3 kernel. The core controller could send and collect signals by its internal different modules. In details, the internal PWM module could produce the driving signal of the ultrasonic probe and the internal AD module could collect the signal received by the other ultrasonic probe. The high clock frequency of the controller and AD module guarantee the accuracy of the data acquisition [5].

\section{Detection Principle}

The sensor detects the liquid level based on the physical phenomenon of the sound intensity attenuation[6-7]. The detection principle is shown in Fig. 2. When the ultrasonic pass through the media inside the dropper, the sound attenuation is associated with liquid level height. The significant difference of the acoustic impedance between liquid and air generates different amplitude of the receiving signal, which is compared with the threshold value. The result of the comparison determines the alarm of the sensor or not.

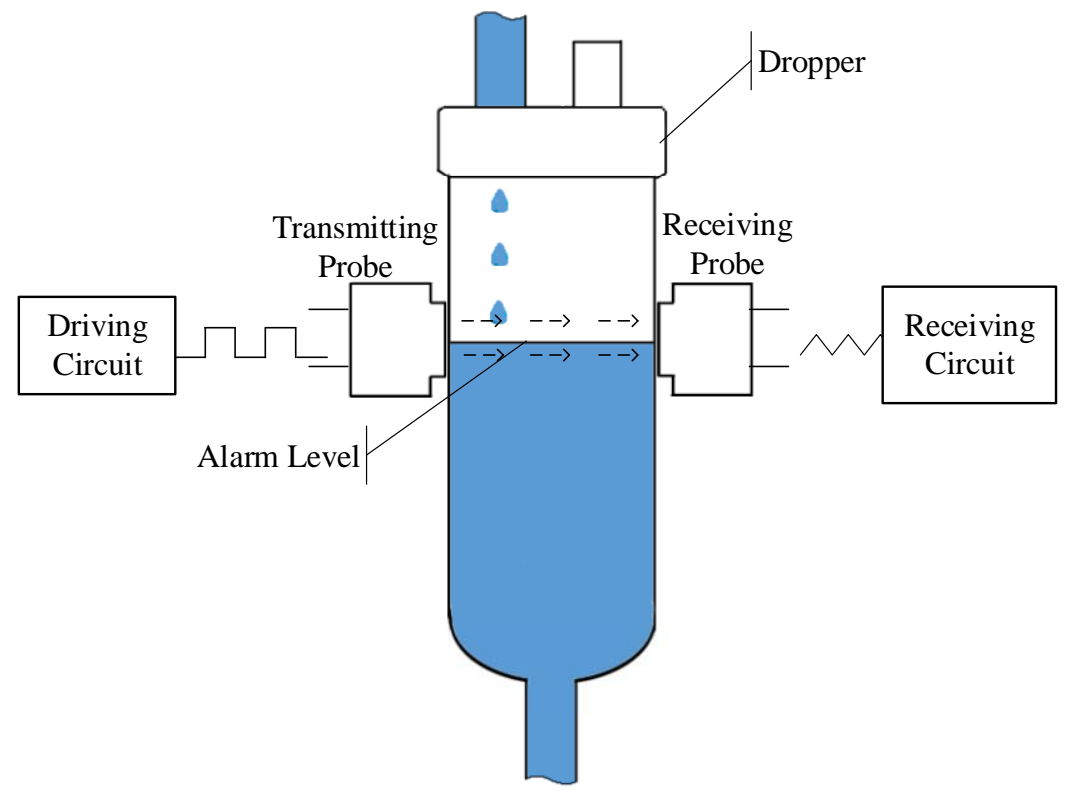

\section{Communication Mode}

Fig.2. Detection principle diagram

Most of the sub-modules of artificial liver use RS-485 bus to communicate with the host computer. And the liquid level sensor communicate with the host computer only, rather than the other sub-modules. Based on this framework, the RS-485 bus and MODBUS protocol which has high reliability and high resistance to the disturbance is used in the sensor to communicate with the host computer[8].

\section{Hardware Design}

The hardware circuit of the sensor consists of the minimum control system, ultrasonic transmission circuit, signal amplification circuit and communication interface. A stable DC supply is necessary for the minimum control system and the sensor uses AMS1117-3.3 to supply 3.3V DC power to STM32F103C8T6.

\section{Transmission Circuit}

The transmission circuit is shown in Fig. 3. The PWM module built-in controller generates $40 \mathrm{KHz}$ square-wave signal which can be converted to RS232 level by MAX232. The output voltage ranges up to DC $18 \mathrm{~V}$ which is large enough to drive the ultrasonic transmitter.

\section{Signal Amplification Circuit}

As is shown in Fig. 4, the signal amplification circuit is based on NE5532. In order to maintain signal integrity and avoid distortion, the quiescent point should be set while NE5532 is single power 
supplied. By adopting resistance divider, the primary voltage is transformed into $2.5 \mathrm{~V}$ to provide the quiescent point of NE5532. The signal collected by ultrasonic receiving probe can be amplified to 11(1+R8/R9) times by amplification circuit for AD acquisition port of STM32.

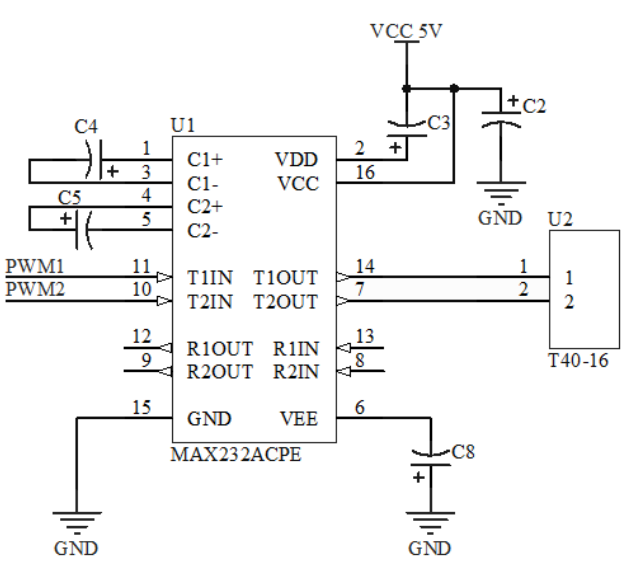

Fig. 3. Transmission circuit

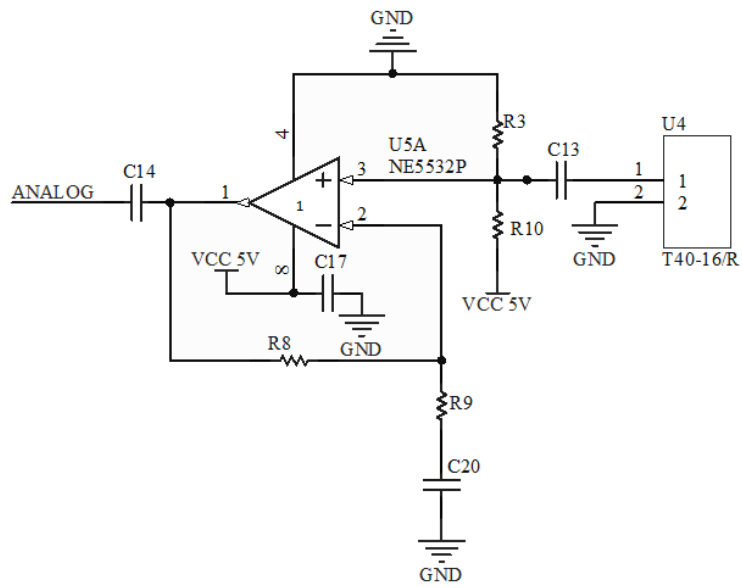

Fig. 4. Signal amplification circuit

\section{Communication Interface Circuit}

The main component of communication interface circuit is the level conversion chip MAX485, which works in half-duplex mode (Fig. 5). A matched resistance of $120 \Omega$ is required at the terminal of the bus so as to absorb the reflected signals on the bus and ensure the accuracy of the data transmitted.

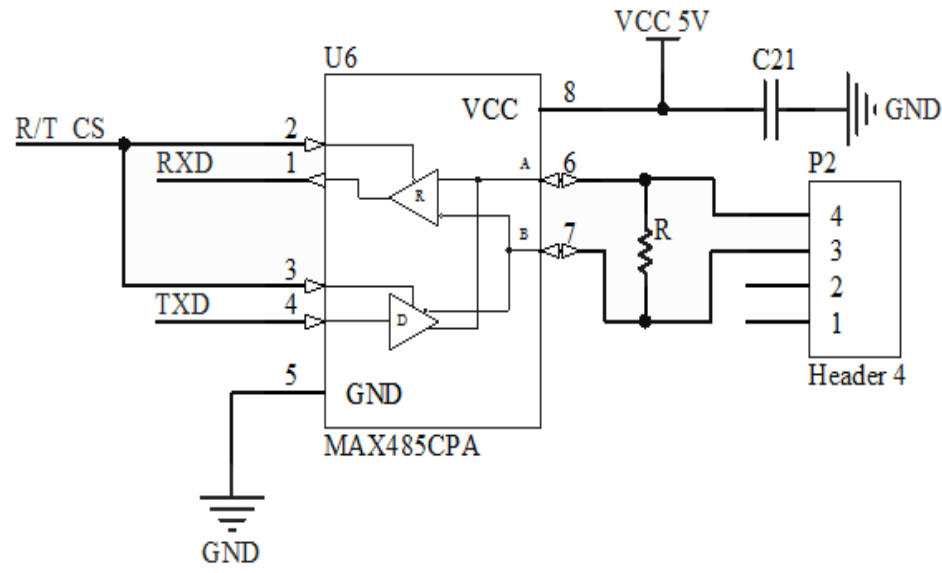

Fig. 5. Communication interface circuit

\section{Software Design}

The software program of the liquid level sensor includes PWM configuration, data acquisition and processing. The clock divide factor of PWM is calculated according to the controller's clock frequency ( $72 \mathrm{MHz}$ ) to generate accurate square-wave signal whose frequency is $40 \mathrm{KHz}$. The signal acquisition and processing procedures directly related to the stability, reliability and sensitivity of the sensor. The control flow chart is shown in Fig. 6.

The frequency of the data acquisition is $400 \mathrm{KHz}$ and the sensor could collect 10 data in each ultrasonic receiving cycle. The controller compares each data $(p v)$ with threshold value (tv) and counts the number $(p t)$, which will be compared with $p n$ afterwards. The value of $p n$ and $t v$ can be modified by MODBUS commands to adjust the sensitivity of the sensor. If the liquid level rise to alarming level, $p t$ will be greater than $p n$ and $w t$ will increase. Once wt is greater than wn in 10 seconds, the sensor will alarm by sending 0x0000 to the host computer through MODBUS protocol Otherwise, the sensor will prompt the host computer by sending 0x0001 about the existence of interference and wt will be set to 0 , and then disable timer interrupt. Such a control mechanism makes 
the sensor stable and reliable. The module address of MODBUS slave and baud rate of the sensor can be modified through the communication interface to enhance the compatibility.

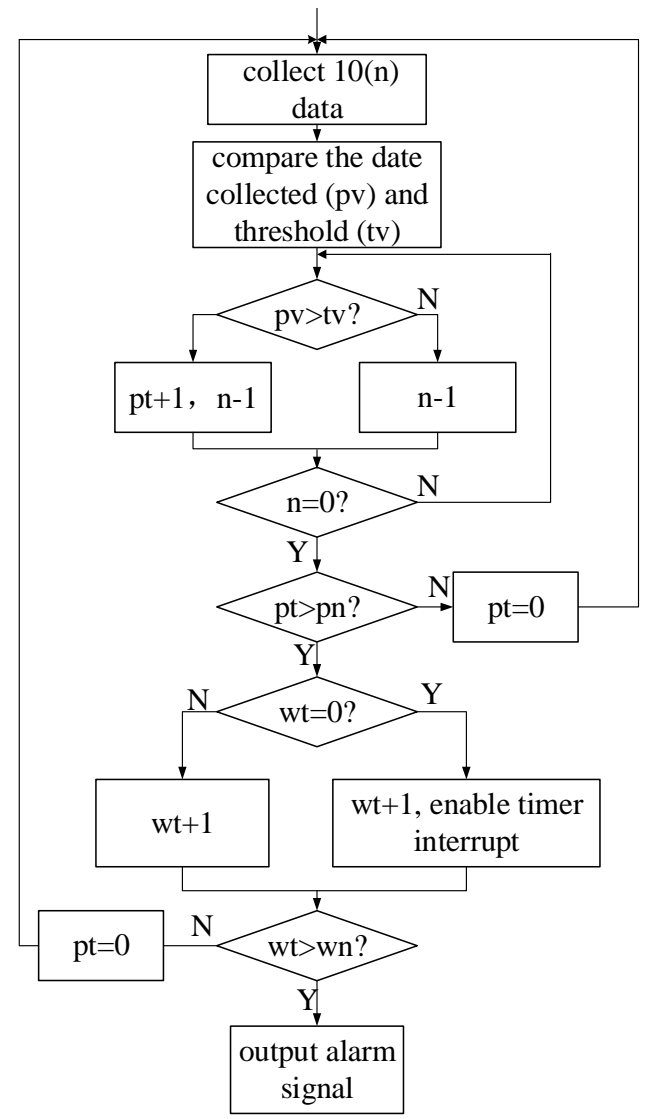

Fig. 6. Control flow chart

\section{Experiments and Results}

Experimental device is shown in Fig. 7. The alarm level is marked on the dropper and a ruler with negative scale is placed beside it. The 0 scale and alarm level should be in the same horizontal and the accuracy of the ruler should be $0.5 \mathrm{~mm}$ at least. And the ultrasonic probes are installed at both sides of the dropper based on the alarm level marked. Liquid is injected into the dropper at the uniform velocity. The serial debugger in the computer will display the message when the sensor alarm and current liquid level will be recorded manually. The line chart in Fig. 8 is made according to 50 times experiments.

The average, standard deviation ( $\sigma$ ) and relative standard deviation $(R S D)$ of the sample data are $0.1116 \mathrm{~mm}, 0.71 \mathrm{~mm}, 2.1 \%$ respectively. The results show that the fluctuation range of the actual alarm level is about $0-1.4 \mathrm{~mm}$ and RSD is less than $5 \%$, which means that the precision of sensor is high. 


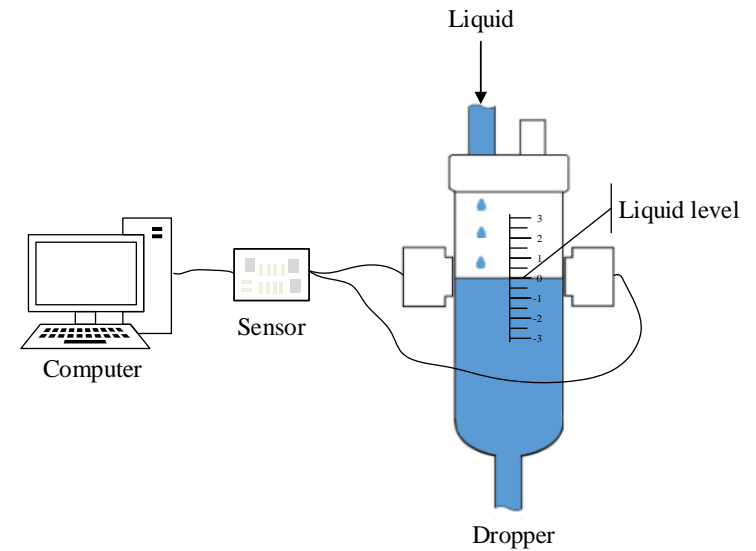

Fig. 7. Experiment device diagram

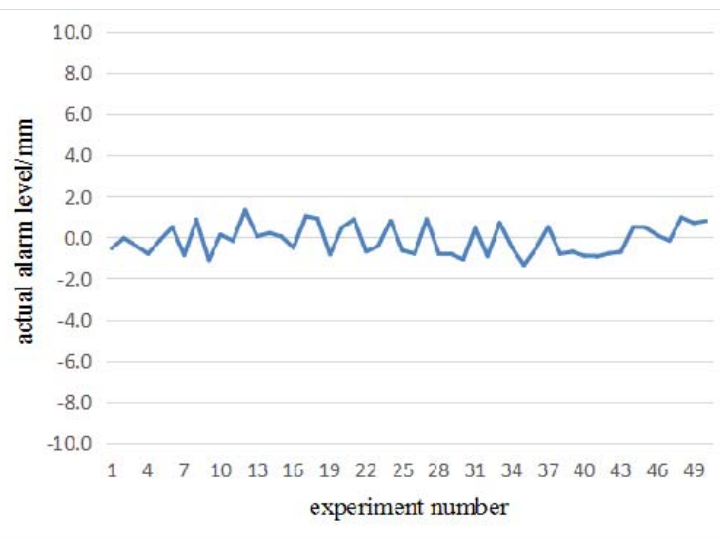

Fig. 8. Experiment data curve

\section{Conclusions}

According to experimental results, the liquid level sensor which combines $40 \mathrm{KHz}$ ultrasonic transceiver probes in this paper has advantages in stability, cost, accuracy and fabrication process. The sensitivity, reliability and interference performance are ensured by software program in STM32. Meanwhile, the short detection cycle of the sensor realizes the real-time transmission of the alarm signal. The sensor can be used in artificial liver system.

\section{Acknowledgements}

This work was financially supported by the Application Research of Bioartificial Liver (2014A030312013) and the Research of Tissue Engineering Construction and Transformation of ZHJ-3 Bioartificial Liver System (2015B020229002).

\section{References}

[1] Lianghong Yin; Daxin Yun; Yeyong Li. Clinical Medicine \& Engineering. 2009, vol. 16, no. 12, 119-123 (in Chinese).

[2] Haiming Zhao; Yinyong Pu; Jichan Wang. Journal of Hunan University of Science \& Technology(Natural Science Edition). 2006, vol. 21, no. 3, 35-38 (in Chinese).

[3] Zhihong Xiao; Xexi Han. Modern Electronics Technique. 2006, vol. 29, no. 19, 97-98,101 (in Chinese).

[4] Chunning Si; Wenke Lu. Instrumentation Technology. 2008, vol. 8, 23-25 (in Chinese).

[5] ST Microelectronics Corporation. STM32F10X Reference manual. 2008.

[6] Wenwei Yao; Xiaojun Xian. PIEZOELECTRICS\&ACOUSTOOPTICS. 2014, vol. 36, no. 4, 548-551,554 (in Chinese).

[7] Robert J. Urick. Principles of Underwater Sound. Newport Beach, Peninsula Pub, 1983, p. 103-107.

[8] Modicon Inc. Modicon Modbus Protocol Reference Guide. 1996. 